\title{
Experimental induction of a perceived "telescoped" limb using a full-body illusion
}

\section{Laura SchmalzI* and H. Henrik Ehrsson}

Brain, Body and Self Laboratory, Department of Neuroscience, Karolinska Institute, Stockholm, Sweden

\section{Edited by:}

Srikantan S. Nagarajan,

University of California at San

Francisco, USA

Reviewed by:

Lutz Jäncke,

University of Zurich, Switzerland

David J. McGonigle,

Cardiff University, UK

Leighton B. Hinkley,

University of California at San

Francisco, USA

\section{*Correspondence:}

Laura Schmalzl, Brain, Body and Self

Laboratory, Department of

Neuroscience, Karolinska Institute,

Retzius Väg 8, 17177 Stockholm,

Sweden.

e-mail: laura.schmalz@ki.se
Phantom limbs refer to the sensation that an amputated or missing limb is still attached to the body. Phantom limbs may be perceived as continuous with the stump so as to resemble a normal limb, or as "telescoped" with the more distal portion of the phantom being perceived as having withdrawn within the stump. Telescoping tends to be related to increased levels of phantom pain, making it a clinically relevant phenomenon to investigate. In the current study we show that a full-body illusion can be used to induce the sensation of a telescoped limb in healthy individuals. For the induction of the full-body illusion, participants saw the body of a mannequin from a first person perspective while being subjected to synchronized visuo-tactile stimulation through stroking. Crucially, the mannequin was missing its left hand so as to resemble an amputee. By manipulating the positioning of the strokes applied to the mannequin's stump with respect to the participants' hand we were able to evoke the sensation of the participants' hand being located either below the stump or, more crucially, "inside" the stump, i.e., telescoped. In three separate experiments these effects were supported by complementary subjective data from questionnaires, verbally reported perceived location of the hand, and manual pointing movements indicating hand position (proprioceptive drift). Taken together our results show that healthy individuals can experience the body of an upper limb amputee as their own, and that this can be associated with telescoping sensations. This is a theoretically important observation as it shows that ownership of an entire body can be evoked in the context of gross anatomical incongruence for a single limb, and that telescoping sensations occur as a consequence of the body representation system trying to reduce this incongruence. Furthermore, the present study might provide a new platform for future studies of the relationship between telescoping and phantom pain in amputees.

Keywords: full-body illusion, body representation, plasticity, phantom limbs, phantom pain, telescoping

\section{INTRODUCTION}

Phantom limbs, that is the sensation that an amputated or missing limb is still attached to the body, are experienced by up to $98 \%$ of amputees (Ramachandran and Hirstein, 1998) and by about 20\% of children with congenital limb aplasia (Melzack et al., 1997). Phantom limbs may be perceived as being intact and continuous with the stump so to resemble a normal limb. In $49-63 \%$ of cases however (Giummarra et al., 2007), phantom limbs are perceived as being "telescoped." That is, the proximal portion of the phantom is perceived to be missing or have shrunken, so that the more distal portion of the phantom is perceived as floating near or "within" the stump (Flor et al., 2006). Telescoping typically begins within the first few weeks post-amputation (Carlen et al., 1978), but the complete extension of the phenomenon may take place over a number of years (Giummarra et al., 2007). It has been proposed that telescoping occurs as a consequence of the fact that the distal portion of any limb is more strongly represented in the cortex relative to its more proximal regions. This proposal is also supported by the fact that lower limbs telescope more rapidly than upper limbs (which are more diffusely represented throughout the cortex due to their integral role in fine motor tasks; Ramachandran and Hirstein, 1998). Telescoping is also a clinically relevant phenomena as it is related to increased levels of phantom limb pain (Flor et al., 2006).
The exact nature of the causal relationship between telescoping and phantom pain remains unclear though. It has been proposed that a contributing factor could be the mismatch between the sensory feedback from the phantom and the cortical regions representing the limb (Flor et al., 2006). That is, the perception of an extended phantom continues to provide sensory feedback to the brain area that previously represented the amputated limb. In contrast, telescoped phantoms activate brain areas remote from the original limb representation, and such cortical reorganization is hypothesized to be the cause of increased levels of phantom pain.

In recent years there has been increasing interest in research investigating the cognitive and neural basis of corporeal awareness and body ownership. One line of research in this area has focused on the empirical investigation of the circumstances under which artificial body parts or even whole bodies can be experienced as ones own. One of the most adopted paradigms in this regard is the rubber hand illusion (RHI), firstly described in modern times by Botvinick and Cohen (1998). In a nutshell, it demonstrates that simultaneous and synchronous stroking of ones unseen hand and an aligned visible rubber hand evokes the illusion that the rubber hand is part of ones own body. More recently, it has also been shown hat the principles underlying the RHI can generalize to whole bodies. Specifically, Petkova and Ehrsson (2008) demonstrated how 
healthy individuals can experience a mannequin's body as their own, if they view it from a first person perspective while being subjected to synchronous visuo-tactile stimulation.

What are the fundamental experimental conditions necessary for these illusions to occur? Firstly, the artificial body or body part have to be seen from a first person perspective and be in a plausible anatomical location and orientation with respect to the real body (Costantini and Haggard, 2007; Petkova and Ehrsson, 2008). Secondly, the tactile stimulation of the real body(part) and the visually perceived stimulation of the artificial body (part) have to occur in temporal and spatial synchrony, allowing for a continuous match between the visual and somatosensory information about the state of the body (Tsakiris and Haggard, 2005; Petkova and Ehrsson, 2008). Thirdly, the artificial body or body part have to have a sufficient degree of "bodily resemblance" (Haans et al., 2008; Petkova and Ehrsson, 2008; Tsakiris et al., 2010). Having said that though, these bodily illusions are actually quite malleable, in the sense that the artificial body(part) does not need to exactly resemble ones own in order for the illusion to occur. For example, Petkova and Ehrsson (2008) reported that both male and female participants experienced the full-body illusion involving a male mannequin to the same degree. Moreover, participants can experience a rubber hand that is much larger or smaller than theirs as their own (Haggard and Jundi, 2009; Bruno and Bertamini, 2010). Finally, people can experience illusory "duplication" of a limb, and feel two right rubber hands as both belonging to their body (Ehrsson, 2009). These observations show that bodily illusions are quite robust, allowing for differences in gender and quite substantial deviations in body shape as well as body size from the real body.

In the current study we explored the robustness, constraints and malleability of the full-body illusion even further. Specifically, we set out to address two main questions: Firstly, whether it is possible to induce a full-body illusion using a mannequin that is missing a hand and whose arm resembles a stump of a hand amputee. That is, can healthy participants experience an amputated body as their own? Secondly, if this is the case, we aimed to explore the possibility of evoking phantom hand sensations by manipulating the perceived position of the participants' hand with respect to the stump of the mannequin. In other words, can the full-body illusion be used to induce the sensation of a "telescoped" limb in healthy individuals? If the full-body illusion allows for such manipulations to be performed, this could have important theoretical as well as clinical implications. From a theoretical point of view, it would allow to further explore the constraints of bodily illusions and the plasticity of body representation. From a clinical perspective, it would represent a platform for the investigation the cognitive and neural mechanisms underlying telescoping in amputees, and potentially even shed light on the relationship between telescoping and phantom pain.

\section{MATERIALS AND METHODS PARTICIPANTS}

Sixteen naive volunteers participated in each of the experiments. Experiment 1: Age: 26-39, Mean = 31.8; Sex: 9 male, 7 female; Handedness: 15 right-handed, 1 left-handed. Experiment 2: Age: 23-66, Mean = 31.8; Sex: 6 male, 10 female; Handedness: 15 righthanded, 1 left-handed. Experiment 3: Age: 22-38, Mean = 27.31; Sex: 7 male, 9 female; Handedness: 15 right-handed, 1 left-handed. Handedness was assessed with the Edinburgh inventory for handedness (Oldfield, 1971). None of the participants had a history of head injury or any other neurological condition, and all had normal (or with contact lenses corrected) vision. The study was approved by the Ethics Committee of the Karolinska Institute, and informed written consent was obtained from all participants.

\section{EXPERIMENTAL SETUP AND BASIC EXPERIMENTAL DESIGN}

The experimental setup and basic experimental design were based on those described by Petkova and Ehrsson (2008).

\section{Head-mounted displays and CCTV cameras}

During all three experiments, participants wore a set of headmounted displays (HMDs; Cybermind Visette Pro PAL, Cybermind Interactive, Maastricht, the Netherlands) with a field of view of $71.5^{\circ}$ and a display resolution of $640 \times 480$. The HMDs were connected to two synchronized color CCTV cameras (Panasonic WV-CP484E), which were attached to a helmet worn by a mannequin, and positioned so as to record the mannequin's body from above (Figure 1). Thus, when looking down toward their own body through the HMDs, participants saw the mannequin's body instead. The cameras were attached side-by side at a distance of $10 \mathrm{~cm}$, with their position corresponding to that of the mannequin's eyes. The images from the left and right video cameras were projected onto the left and right
A

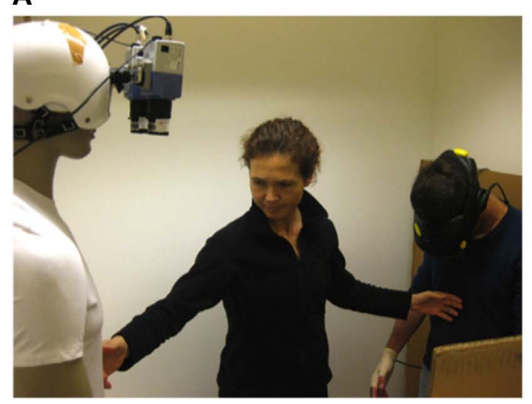

B

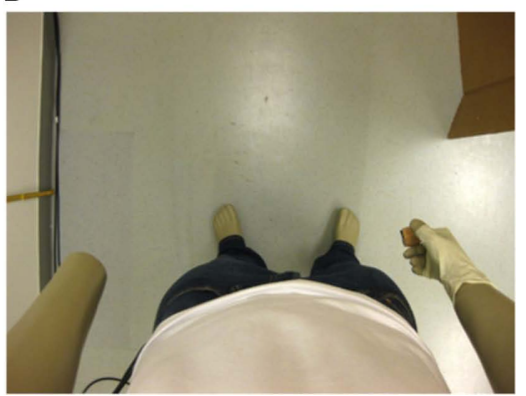

FIGURE 1 | Experimental setup. (A) For the induction of the full-body illusion synchronous strokes were applied to the participants and the mannequin, while the participants were viewing the mannequin's body through a set of HMDs. (B) View of the mannequin's body, which was missing its left hand, from the participants' perspective. 
side of the HMDs respectively, allowing for a true stereoscopic image. In addition, the CCTV camera signals were directly relayed into the HMDs without any external conversion devices, hence participants viewed the recordings with no noticeable delay.

\section{Mannequin}

A male mannequin, dressed with blue jeans and a white T-shirt, was used for both male and female participants in all experiments. This choice was based on the fact that Petkova and Ehrsson (2008) did not find any gender effects in any of their full-body illusion experiments. Crucially for the experimental manipulations described below, in our setup the mannequin was missing its left hand. Hence, the mannequin's left arm resembled a "stump" of left hand amputees (Figure 1).

\section{Induction of the full-body illusion}

Participants wore the HMDs connected to CCTV cameras as described above. They assumed a standing position and were asked to tilt their head forward as if they were looking down at their own body, so that they viewed the mannequin's body from a first person perspective. In order to induce a full-body illusion, namely the experience of perceiving the mannequin's body as one's own, participants were then subjected to synchronized visuo-tactile stimulation through stroking. That is, the experimenter simultaneously stroked the participants' body (which was out of view) and the mannequin's body (which was in full view). Hence, participants experienced the visual stimulation seen on the mannequin corresponding to the tactile stimulation felt on their own bodies. In all three experiments, stroking was initially performed on the torso and the right hand (to induce the full-body illusion), and subsequently on the left hand (to induce the specific effects of each experimental condition). Details about the exact duration and characteristics of the stroking in each of the experimental conditions are provided below.

\section{EXPERIMENT 1}

\section{Stroking of the torso and right hand - induction of the full-body} illusion

The induction of the full-body illusion was performed by a 1-min stroking session of the torso, followed by a 1 -min stroking session of the right forearm and hand. Approximately 60 strokes were applied per minute, and the stroke length varied from about $1-5 \mathrm{~cm}$. An irregular pattern of stroking was used to avoid expectations about the timing of the visuo-tactile stimulation events in the participants.

\section{Stroking of the left hand - induction of specific experimental effects}

Stroking of the torso and the right hand was followed by a 1-min session of simultaneous stroking of the participants' left hand and the mannequin's stump. As above, strokes were always applied in temporal synchrony. With regard to the spatial location of the strokes however, there were two different experimental conditions: (1) Simultaneous stroking of the participant's hand and the empty space below the mannequin's stump in spatially corresponding locations, so that there was an exact overlap of the strokes felt on the unseen hand and the strokes seen in empty space below the mannequin's stump; in this mode of stimulation the participants' wrist coincided with the end of the mannequin's stump. (2) Simultaneous stroking of the participant's hand and the end of the mannequin's stump, so that the felt and seen strokes occurred with an offset of approximately $15 \mathrm{~cm}$; in this mode of stimulation the participants' fingertips coincided with the end of the mannequin's stump (Figure 2). In Condition 1, the strokes applied to the empty space below the mannequin's stump were carefully monitored so as to resemble the shape and size of each participant's hand as closely as possible. In Condition 2, particular emphasis was given on simultaneously touching the participants' fingertips and the very end of the mannequin's stump. Participants experienced each condition once in counterbalanced order. In between each condition the HMDs were removed, and participants were asked to fill out a questionnaire in regard to their experience during the stroking (see below).

\section{Questionnaire}

Following each stroking session, consisting of a total of 3-min stroking of the torso, the right hand and then the left hand (Condition 1 or 2), participants were asked to complete a questionnaire. The questionnaire consisted of 12 statements describing possible perceptual effects that might be elicited by the stroking, and participants were asked to affirm or deny experiencing each
A

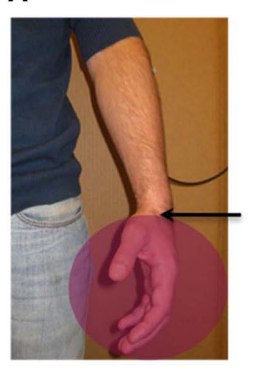

Condition 1

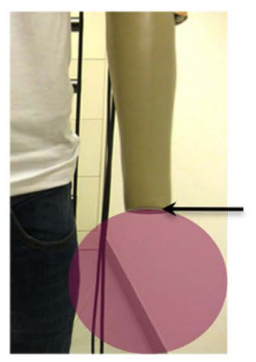

FIGURE 2 | Stroking conditions. (A) Condition 1: Simultaneous stroking of the participant's hand and the empty space below the mannequin's stump, so as to make the participant's wrist coincide with the end of the mannequin's stump. (B) Condition 2: Simultaneous stroking of the participant's hand and the end of the
B Condition 2

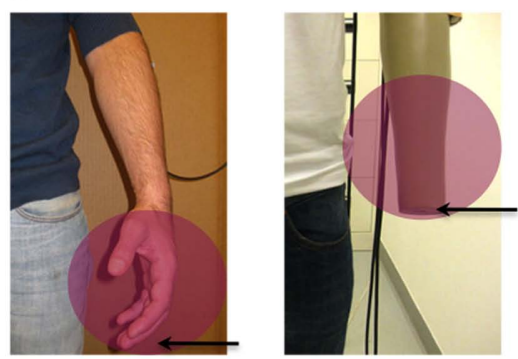

mannequin's stump, so as to make the participant's fingertips coincide with the end of the mannequin's stump. The red circles indicate the area on which simultaneous strokes were applied, and the arrows indicate the point of the arm of the participant that was touched at the same time as the end of the mannequin's stump. 
effect on a seven-point Likert scale. Two statements were intended to capture the overall illusory experience of perceiving the mannequin's body as one's own, two statements were intended to capture the perceived location of the left hand expected to occur in each condition, and one statement referred to the perceived length of the left arm. For each of these statements intending to capture illusory sensations, there was a control statement aimed at capturing the participants' suggestibility and task compliance. Lastly, two remaining statements referred to sensations of pain, numbness, burning or pins and needles, which we thought could potentially be experienced by participants during the illusion (Figure 3). The order of the questions was randomized across participants to avoid any order effects. The crucial measures of interest were whether participants reported to have experienced the full-body illusion during the stroking sessions, and if they did whether they perceived their left hand to be located in a different position with respect to the stump of the mannequin in Condition 1 vs. Condition 2.

\section{EXPERIMENT 2 \\ Stroking of the torso and right hand - induction of the full-body illusion}

The induction of the full-body illusion was performed following the same procedure as in Experiment 1, except that the stroking of the torso as well as the stroking of the right forearm and hand were shortened to $30 \mathrm{~s}$. The decision to shorten the stroking duration was taken following subjective reports of participants in Experiment 1 , indicating that halving the stroking duration would still be sufficient to induce the full-body illusion.

\section{Stroking of the left hand - induction of specific experimental effects}

Stroking of the left hand was performed following the exact same procedure as described in Experiment 1 for both Condition 1 and Condition 2. Participants experienced each condition three times in a counterbalanced order. At the end of each stroking session, participants performed a visual judgment task (see below).

\section{Visual judgment task}

After each stroking session, consisting of a total of 2-min stroking of the torso, the right hand and then the left hand (Condition 1 or 2), participants were asked to perform a visual judgment task in which they had to visually judge the perceived location of their left fingertips. The purpose of this task was to obtain a objective measure of the experimental effect found in Experiment 1, namely the difference in the perceived position of the left hand in Condition 1 (below the stump) vs. Condition 2 ("inside" the stump). The task was performed as follows: After each stroking session participants were asked to look at "their" left arm, that is the left stump of the mannequin, through the HMDs. Then, the experimenter moved a ruler from the floor up toward the stump, and participants verbally instructed the experimenter to adjust the height of the ruler to the perceived location of their left fingertips. The absolute height of the position was then manually recorded by the experimenter on a measuring band attached to the wall next to which the participant was standing (measurements were rounded up to $0.5 \mathrm{~cm}$ ). A spirit level ruler was used in order to assure the its horizontal alignment when recording the height on the measurement band (Figure 4). The crucial question of interest was whether there would be a significant difference in the visually judged height

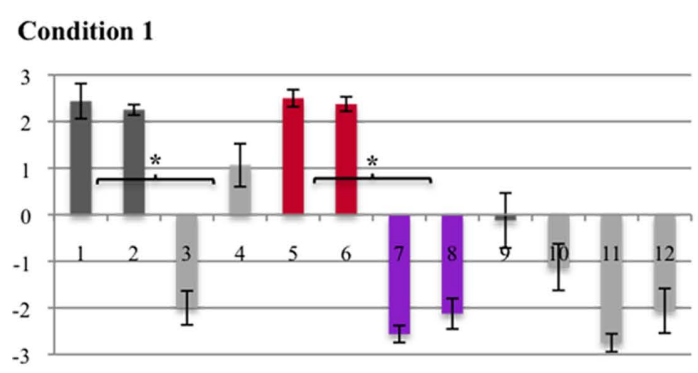

Condition 2

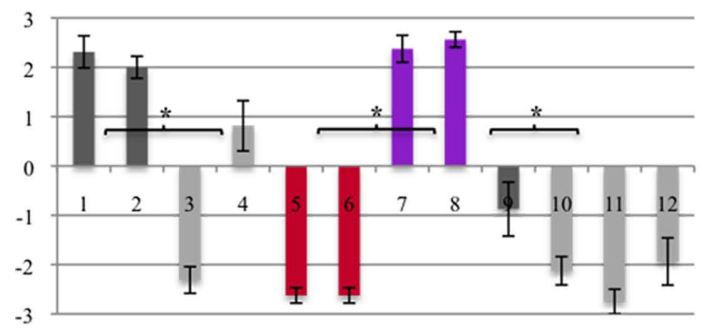

FIGURE 3 | Experiment 1 - Questionnaire. The questionnaire consisted of 12 statements referring to differential aspects of the perceptual experience induced by the stroking sessions: Experience of the full-body illusion (Statements 1-4); Perceived location of the left hand (Statements 5-8); Perceived length of the left arm (Statements 9-10); Sensations of pain, numbness, burning or pins and needles, which could potentially be experienced
1. It seemed as if I could feel the touch given to the mannequin

2. It felt like the mannequin's body was my own body

3. It felt as if I had two bodies

4. The mannequin's body began to resemble my own body in terms of shape, skin tone, or some other visual feature

5. It felt as if my left hand was located below the stump of the mannequin

6. I could feel my left wrist to be in the same location as the end of the stump of the mannequin

7. It felt as if my left hand was located "inside" the stump of the mannequin

8. I could feel my left fingers to be in the same location as the end of the stump of the mannequin

9. It felt as if my left arm was shorter than my right arm

10. It felt as if my left arm was lighter than the right arm

11. I felt pain in my left hand

12. I felt sensations such as numbness, burning or pins \& needles in my left hand

by participants during the illusion (Statements 11-12). Participants were asked to affirm or deny each statement on a seven-point Likert scale (+3 = strongly agree; -3 = strongly disagree) - the graph shows the mean group values with standard error bars. Wilcoxon signed rank tests were performed between illusion statements and control statements, as well as between Condition 1 and Condition 2 - for more details see section "Methods and Results." * $p<0.05$. 
of the perceived position of the left fingertips following the two stroking conditions, reflecting the subjectively reported difference observed in Experiment 1.

\section{Questionnaire}

Following the six experimental sessions involving stroking and the visual judgment task, participants were given a short break during which the HMDs were removed. Then, the experimental setup was resumed, and participants experienced each stroking condition one more time in a counterbalanced order. After each condition, participants were asked to complete a shortened version of the questionnaire used in
Experiment 1 (Figure 5). As in Experiment 1, the crucial measures of interest were whether participants reported to have experienced the full-body illusion during the stroking sessions, and if they did whether they perceived their left hand to be located in a different position with respect to the stump of the mannequin in Condition 1 vs. Condition 2.

\section{EXPERIMENT 3}

Stroking of the torso and right hand - induction of the full-body illusion

The induction of the full-body illusion was performed following the exact same procedure as in Experiment 2.
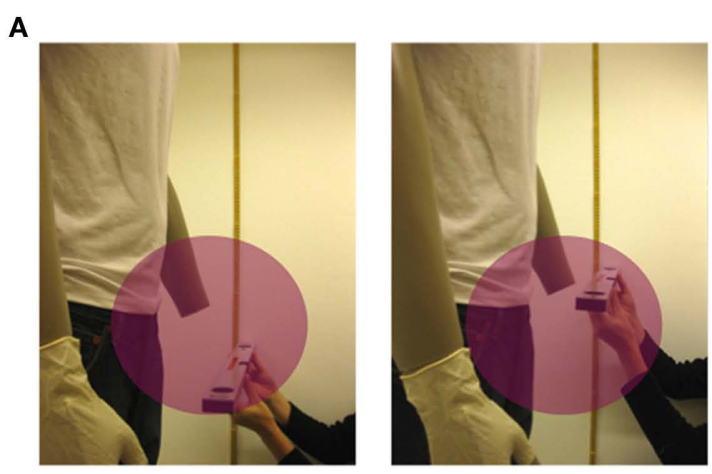

B Perceived height of fingertips

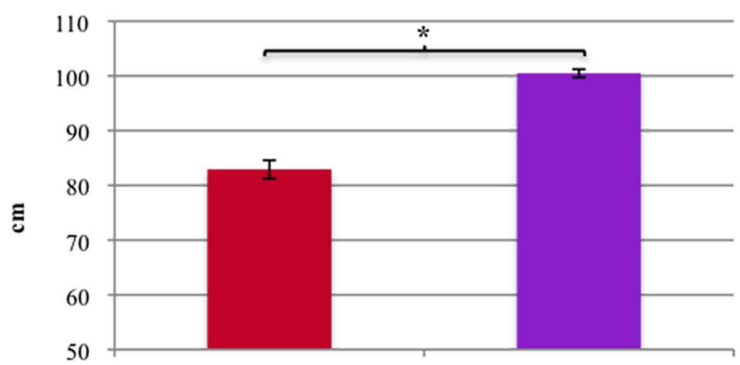

$\mathrm{IH}$

Condition

TH

FIGURE 4 | Experiment 2 -Visual judgment task. (A) At the end of each stroking session participants were asked to judge the perceived height of their fingertips by verbally instructing the experimenter to adjust the height of a ruler. (B) Mean height of the perceived position of the finger tips in the $\mathrm{IH}$ and TH condition (paired samples $t$-test $-p<0.001$.
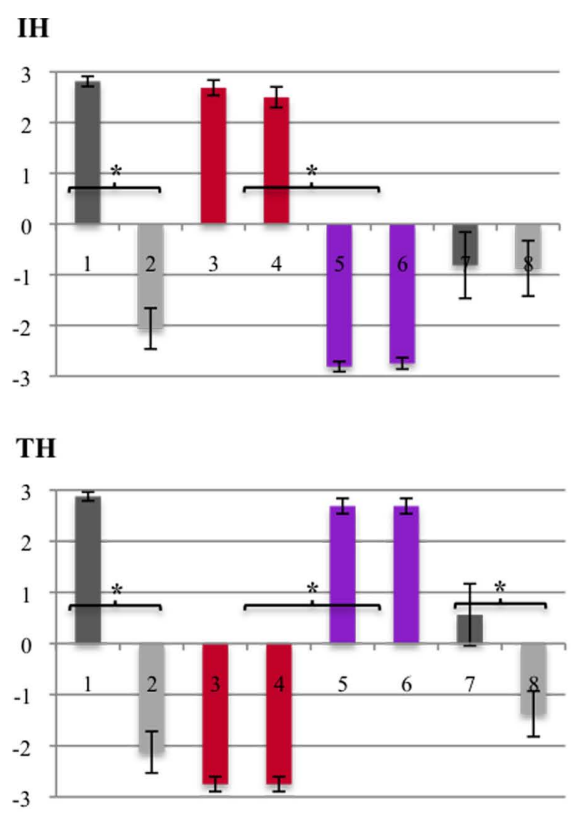

1. It seemed as if I could feel the touch given to the mannequin

2. It felt as if I had two bodies

3. It felt as if my left hand was located below the stump of the mannequin

4. I could feel my left wrist to be in the same location as the end of the stump of the mannequin

5. It felt as if my left hand was located "inside" the stump of the mannequin

6. I could feel my left fingers to be in the same location as the end of the stump of the mannequin

7. It felt as if my left arm was shorter than my right arm

8. It felt as if my left arm was lighter than the right arm
FIGURE 5 | Experiment 2 - Questionnaire. Shortened version of the questionnaire used in Experiment 1 consisting of eight statements: Experience of the full-body illusion (Statements 1-2); Perceived location of the left hand (Statements 3-6); Perceived length of the left arm (Statements 7-8); Participants were asked to affirm or deny each statement on a seven-point Likert scale
(+3 = strongly agree; -3 = strongly disagree) - the graph shows the mean group values with standard error bars. Wilcoxon signed rank tests were performed between illusion statements and control statements, as well as between the $\mathrm{IH}$ and TH conditions - for more details see section "Methods and Results." ${ }^{*} p<0.05$. 


\section{Stroking of the left hand - induction of specific experimental effects} Stroking of the left hand was performed following the exact same procedure as described in the previous two experiments for both Condition 1 and Condition 2. Again, participants experienced each condition three times in a counterbalanced order. Before and after each stroking session, participants performed a pointing task (see below).

\section{Pointing task}

Before and after each stroking session, consisting of a total of 2-min stroking of the torso, the right hand and then the left hand (Condition 1 or 2), participants were asked to perform a pointing task in which they had to point to the perceived position of their left thumb. The purpose of this task was to obtain an objective motoric measure of the proprioceptive drift of the left hand in Condition 1 (when the hand is perceived to be below the stump) vs. Condition 2 (when the hand is perceived to be "inside" the stump). In contrast to the visual judgment task, during the pointing movements all visual cues were removed, so that the proprioceptive drift measure would reflect an effect based on proprioceptive information alone. The setup and procedure of the pointing task were as follows: Pointing movements were recorded using a FASTRAK electromagnetic motion tracking system (Polhemus, Colchester, VT, USA). The FASTRAK system consists of a transmitter and two small sensors, and allows the tracking of the position $(x, y$, and $z$ Cartesian coordinates) and orientation (azimuth, elevation, and roll) of the sensors with respect to the transmitter as they move through space. During the movement data is recorded at a frequency of $60 \mathrm{~Hz}$. Sensor 1 was placed on the participants' right index finger, and used to track the pointing movement. Sensor 2 was placed below the participants' left elbow, and used to monitor the participant's position in space during the experiment. In addition to the FASTRAK equipment, a plexiglass board was placed on the inside of the participants' left hand, and used to provide a constant horizontal end point for the pointing movement. Participants were positioned so that their left hand was placed at a distance of about $10 \mathrm{~cm}$ from the plexiglass board, and the height of their thumb was marked on the board so that they could be adjusted to the exact same position prior to each stroking session. Then, before and after each stroking session, participants were asked to point toward the tip of their left thumb with their right index finger, by continuing to move until their right index finger touched the plexiglass board (Figure 6). During all pointing movements, the participants' vision was occluded (by temporarily cutting the electricity supply to the CCTV cameras) in order to exclude any reliance on visual reference points to guide the pointing movements. The crucial question of interest was whether the difference in perceived hand position in Condition 1 vs. Condition 2 observed in the previous experiments, would be reflected by a difference in pointing before vs. after the stroking sessions in the respective conditions.

\section{Questionnaire}

Following the six experimental sessions involving stroking and the pointing task, participants were given a short break during which the HMDs were removed. Then, the experimental setup was resumed, and participants experienced each stroking condition one more time in a counterbalanced order. After each condition, participants were asked to complete the same questionnaire as the one used in Experiment 2 (Figure 7). Again, the crucial measures of interest were whether participants reported to have experienced the full-body illusion during the stroking sessions, and if they did whether they perceived their left hand to be located in a different position with respect to the stump of the mannequin in Condition 1 vs. Condition 2.

\section{DATA ANALYSIS}

All the data acquired in the three experiments was tested for normal distribution using the Shapiro-Wilk test. The data obtained from the questionnaires did not pass the test for normality in any of the experiments, hence non-parametric statistics were used for all analyses of questionnaire data. In contrast, the data obtained from the visual judgment task and the pointing task did pass the test for normality, hence parametric statistics were used for the analysis of proprioceptive drift measures. All analyses were based on a priory hypotheses, and hence consisted of planned comparisons. For questionnaire data, comparisons were performed between the illusion questions and their respective control questions, or between the
A

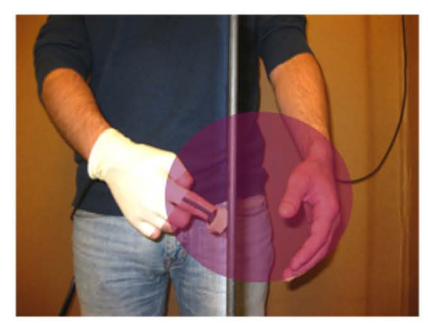

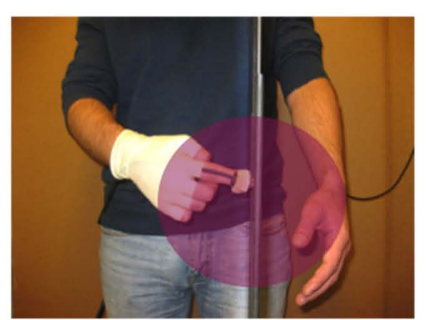

B

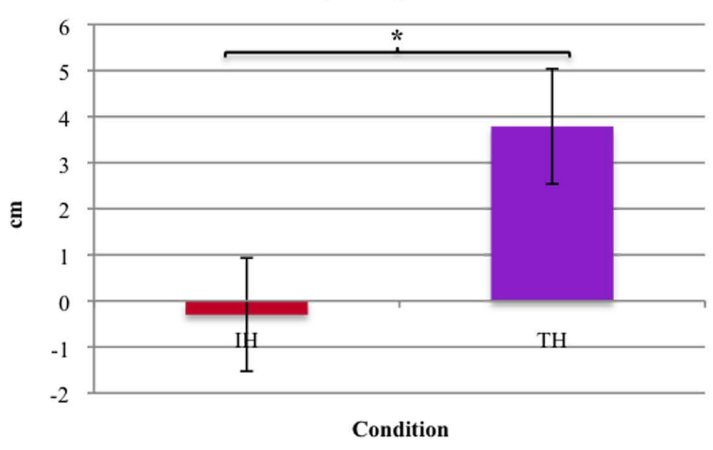

FIGURE 6 | Experiment 3 - Pointing task. (A) Before and after each stroking session participants were asked to point toward the tip of their left thumb with their right index finger. A sensor (right index finger) was used to measure the pointing movement and a second sensor (left elbow) was used to monitor the participant's position in space during the experiment. Cameras were occluded during each pointing movement to exclude reliance on visual information. (B) Proprioceptive drift for the $\mathrm{IH}$ and $\mathrm{TH}$ condition as measured by the difference in pointing before vs. after the induction of the illusion (paired samples $t$-test $-p<0.001$ ). 


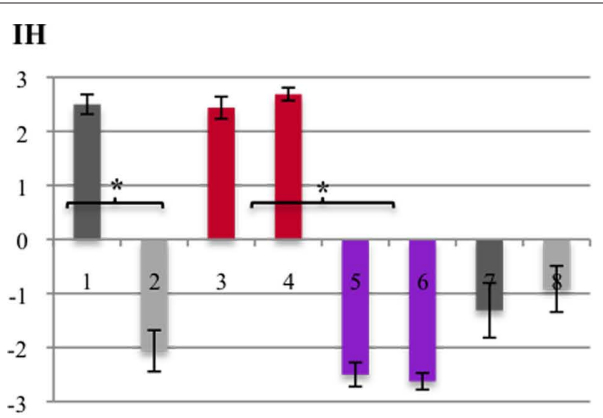

TH

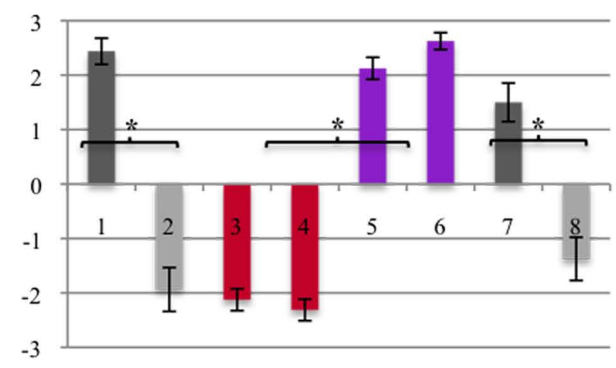

1. It seemed as if I could feel the touch given to the mannequin

2. It felt as if I had two bodies

3. It felt as if my left hand was located below the stump of the mannequin

4. I could feel my left wrist to be in the same location as the end of the stump of the mannequin

5. It felt as if my left hand was located "inside" the stump of the mannequin

6. I could feel my left fingers to be in the same location as the end of the stump of the mannequin

7. It felt as if my left arm was shorter than my right arm

8. It felt as if my left arm was lighter than the right arm
FIGURE 7 | Experiment 3 - Questionnaire. Shortened version of the questionnaire used in Experiment 1 consisting of eight statements: Experience of the full-body illusion (Statements 1-2); Perceived location of the left hand (Statements 3-6); Perceived length of the left arm (Statements 7-8); Participants were asked to affirm or deny each statement on a seven-point Likert scale
(+3 = strongly agree; -3 = strongly disagree $)$ - the graph shows the mean group values with standard error bars. Wilcoxon signed rank tests were performed between illusion statements and control statements, as well as between the $\mathrm{IH}$ and TH conditions - for more details see section "Methods and Results." ${ }^{*} p<0.05$. two stroking conditions (Condition 1 and Condition 2 described above), using Wilcoxon signed rank tests. For proprioceptive drift measures, comparisons were performed between the two stroking conditions using paired samples $t$-tests. Since all analyses were based on a priory hypotheses, no post hoc corrections for multiple comparisons were performed. For all analyses, alpha was set to 0.05.

\section{RESULTS \\ EXPERIMENT 1 \\ Aim}

Experiment 1 had two main aims. Firstly, we aimed to investigate whether it is possible to induce a full-body illusion involving the use of a mannequin that is missing a limb, that is whether participants would accept a body resembling that of a hand amputee as their own. Secondly, if this is the case, we aimed to investigate whether during the full-body illusion it is possible to manipulate the perceived location of the participants' hand with respect to the stump of the mannequin.

\section{Questionnaire results}

As mentioned in section "Materials and Methods," the statements of the questionnaire referred to three distinct aspects of the perceptual experience induced by the stroking sessions: (a) Experience of the full-body illusion, (b) Perceived location of the left hand, and (c) Perceived length of the left arm. The comparison between illusion and control statements, as well as the comparisons between stroking conditions for each of these aspects was therefore performed independently (Figure 3). (a) Experience of the full-body illusion (Illusion statements 1-2, Control statements 3-4)

Following both Condition 1 and Condition 2, participants clearly affirmed experiencing the full-body illusion (Illusion statements mean score vs. control statements mean score - Wilcoxon signed rank test: Condition 1: $Z=-3.481$, $p<0.001$; Condition 2: $Z=-3.521, p<0.001)$. There was no difference in the perceived strength of the full-body illusion in the two conditions (Condition 1 vs. Condition $2-$ Wilcoxon signed rank test: Illusion statements mean score: $Z=-1.261, p=0.207$; Control statements mean score: $Z=-1.628, p=0.103)$.

(b) Perceived location of the left hand illusion (For Condition 1: Illusion statements 5-6, Control statements 7-8; For Condition 2: Illusion statements 7-8, Control statements 5-6)

There was a significant difference in the perceived position of the left hand in the two stroking conditions. In Condition 1, participants clearly affirmed that they perceived their hand to be located below the stump, with their wrist being in the same position as the end of the stump of the mannequin (Illusion statements mean score vs. control statements mean score - Wilcoxon signed rank test: $Z=-3.532, p<0.001)$. In Condition 2, participants clearly affirmed that they perceived their hand to be located "inside" the stump, with their left fingertips being in the same position as the end of the stump of the mannequin (Illusion statements mean score vs. control statements mean score - Wilcoxon signed rank test: 
$Z=-3.551, p<0.001)$. When comparing the participants' responses between Conditions 1 and 2, there was a significant difference for both the mean score of the statements referring to the hand being perceived below the stump (Wilcoxon signed rank test: $Z=-3.544, p<0.001$ ), and the mean score of the statements referring to the hand being perceived "inside" the stump (Wilcoxon signed rank test: $Z=-3.533, p<0.001$ ).

(c) Perceived length of the left arm (Illusion statement 9, Control statement 10)

Participants did not affirm experiencing their left arm to be shorter than their right arm in either of the conditions. However, participants responded more positively to the illusion statement (i.e., my left arm felt shorter) than to the control statement (i.e., my left arm felt lighter) in Condition 2 (Wilcoxon signed rank test: $Z=-2.205, p=0.027$ ), whereas this was not the case in Condition 1 (Wilcoxon signed rank test: $Z=-1.711, p=0.087)$.

(d) Sensations of pain, numbness, burning or pins and needles (Statements 11-12)

Participants did not affirm experiencing any pain or sensations such as numbness, burning or pins and needles in either of the conditions.

\section{EXPERIMENT 2}

\section{Aim}

The main aim of Experiment 2 was to obtain a more objective measure of the experimental effect found in Experiment 1, namely the difference in the perceived position of the left hand in Condition 1 (below the stump) vs. Condition 2 ("inside" the stump). Specifically, the aim was to investigate whether there would be a significant difference in the visually judged height of the perceived position of the left fingertips following the two stroking conditions. Given the results of the subjective reports in Experiment 1 , hereafter Condition 1 will be referred to as "invisible hand condition" (IH) and Condition 2 will be referred to as "telescoped hand condition" (TH).

\section{Visual judgment task results}

The results of the visual judgment task showed a significant difference in the visually judged height of the perceived position of the left fingertips in the TH vs. IH condition (paired samples $t$-test: $t=20.800, p<0.001$; Figure 4).

\section{Questionnaire results}

As in Experiment 1, the comparison between illusion and control statements, as well as the comparisons between stroking conditions for each of the aspects addressed by the questionnaire was performed independently 1 (Figure 5).

(a) Experience of the full-body illusion (Illusion statement 1, Control statement 2)

As in Experiment 1, following both the $\mathrm{IH}$ and $\mathrm{TH}$ conditions, participants clearly affirmed experiencing the fullbody illusion (Illusion vs. control statement - Wilcoxon signed rank test: $\mathrm{IH}: Z=-3.496, p<0.001$; TH: $Z=-3.618$, $p<0.001)$. Also, there was no difference in the perceived strength of the full-body illusion in the two conditions (IH vs.
$\mathrm{TH}$ - Wilcoxon signed rank test: Illusion statement: $Z=-0.577, p=0.564$; Control statement: $Z=-0.276$, $p=0.783$.

(b) Perceived location of the left hand illusion (For Condition 1: Illusion statements 3-4, Control statements 5-6; For Condition 2: Illusion statements 5-6, Control statements 3-4)

Again, the participants' responses mirrored those of Experiment 1 . In the IH condition, participants clearly affirmed that they perceived their hand to be located below the stump, with their wrist being in the same position as the end of the stump of the mannequin (Illusion statements mean score vs. control statements mean score - Wilcoxon signed rank test: $Z=-3.591, p<0.001)$. In the TH condition, participants clearly affirmed that they perceived their hand to be located "inside" the stump, with their left fingertips being in the same position as the end of the stump of the mannequin (Illusion statements mean score vs. control statements mean score - Wilcoxon signed rank test: $Z=-3.653, p<0.001$ ). When comparing the participants' responses between the $\mathrm{IH}$ and $\mathrm{TH}$ conditions, there was a significant difference for both the mean score of the statements referring to the hand being perceived below the stump (Wilcoxon signed rank test: $Z=-3.575, p<0.001$ ), and the mean score of the statements referring to the hand being perceived "inside" the stump (Wilcoxon signed rank test: $Z=-3.654, p<0.001$ ).

(c) Perceived length of the left arm (Illusion statement 7, Control statement 8)

Compared to Experiment 1, participants showed an increased tendency to affirm experiencing their left arm as being shorter than their right arm in the $\mathrm{TH}$ condition. However, the average response (although above 0) did not differ significantly from that in the IH condition (Wilcoxon signed rank test: $Z=-1.450, p=0.147)$. As in Experiment 1 though, participants responded more positively to the illusion statement (i.e., my left arm felt shorter) than to the control statement (i.e., my left arm felt lighter) in the TH condition (Wilcoxon signed rank test: $Z=-2.271, p=0.023$ ), whereas this was not the case in the IH condition (Wilcoxon signed rank test: $Z=-0.360, p=0.719)$.

\section{EXPERIMENT 3 \\ Aim}

As in Experiment 2, the main aim of Experiment 3 was to obtain an objective measure of the proprioceptive drift of the left hand in the $\mathrm{IH}$ vs. the $\mathrm{TH}$ conditions. In contrast to the visual judgment task however, in this experiment participants had to manually indicate the perceived location of their left hand with no available visual cues.

\section{Pointing task results}

The proprioceptive drift was measured by the difference of the height to which participants pointed before vs. after the stroking sessions in the TH as well as the IH conditions. In line with our prediction, the proprioceptive drift, measured by the difference of the position of Sensor 1, was significantly larger in the TH condition compared to the IH condition (paired samples $t$-test: $t=-4.086$, 
$p<0.001)$. We further used one sample $t$-tests to evaluate whether the drift was significantly different from 0 when considering the data from each condition separately. The results showed a significant proprioceptive drift in the TH condition $(t=-7.305, p<0.001)$ but not in the IH condition $(t=0.997, p=0.344)$. Importantly, no such differences was observed for Sensor 2, excluding that the proprioceptive drift in the TH condition was simply caused by the participants moving during the experiment (paired samples $t$-test: $t=-0.196, p=0.136$; Figure 6).

\section{Questionnaire results}

As in the previous experiments, the comparison between illusion and control statements, as well as the comparisons between stroking conditions for each of the aspects addressed by the questionnaire was performed independently (Figure 7).

(a) Experience of the full-body illusion (Illusion statement 1, Control statement 2)

Again, participants clearly affirmed experiencing the fullbody illusion in both the $\mathrm{IH}$ and $\mathrm{TH}$ conditions (Illusion vs. control statement - Wilcoxon signed rank test: IH: $Z=-3.570, p<0.001$; TH: $Z=-3.495, p<0.001)$. The perceived strength of the full-body illusion did also not differ in the two conditions ( IH vs. TH - Wilcoxon signed rank test: Illusion statement: $Z=-0.276, p=0.783$; Control statement: $Z=-0.447, p=0.655)$.

(b) Perceived location of the left hand illusion (For Condition 1: Illusion statements 3-4, Control statements 5-6; For Condition 2: Illusion statements 5-6, Control statements 3-4)

The participants' responses replicated those of the previous experiments. In the IH condition, participants clearly affirmed that they perceived their hand to be located below the stump, with their wrist being in the same position as the end of the stump of the mannequin (Illusion statements mean score vs. control statements mean score - Wilcoxon signed rank test: $Z=-3.570, p<0.001)$. In the TH condition, participants clearly affirmed that they perceived their hand to be located "inside" the stump, with their left fingertips being in the same position as the end of the stump of the mannequin (Illusion statements mean score vs. control statements mean score - Wilcoxon signed rank test: $Z=-3.495, p<0.001)$. When comparing the participants' responses between the $\mathrm{IH}$ and $\mathrm{TH}$ conditions, there was a significant difference for both the mean score of the statements referring to the hand being perceived below the stump (Wilcoxon signed rank test: $Z=-3.526, p<0.001$ ), and the mean score of the statements referring to the hand being perceived "inside" the stump (Wilcoxon signed rank test: $Z=-3.533, p<0.001$ ).

(c) Perceived length of the left arm (Illusion statement 7, Control statement 8)

Contrary to the previous experiments, participants affirmed experiencing their left arm as being shorter than their right arm in the $\mathrm{TH}$ condition, with the average rating differing significantly from that of in the $\mathrm{IH}$ condition (Wilcoxon signed rank test: $Z=-3.060, p=0.002$ ). As in the previous experiments, participants responded more positively to the illusion statement (i.e., my left arm felt shorter) than to the control statement (i.e., my left arm felt lighter) in the TH condition (Wilcoxon signed rank test: $Z=-2.983, p=0.003$ ), whereas this was not the case in the IH condition (Wilcoxon signed rank test: $Z=-0.413, p=0.679$ ).

\section{DISCUSSION}

The current study demonstrates that the sensation of a telescoped hand can be induced experimentally in healthy individuals using a full-body illusion. Questionnaire data across all three experiments showed that participants experienced an ownership illusion of an artificial amputee body. That is, independently of the stroking condition, participants experienced the mannequin's body as their own even if it was missing a hand. Questionnaire data also showed that, depending on the stroking condition, participants affirmed experiencing their left hand to be located either below ("invisible hand") or inside the stump of the mannequin ("telescoped hand"). These latter perceptual phenomena were confirmed by objective measures of proprioceptive drift in both a visual judgment task (Experiment 2) and a pointing task (Experiment 3).

Our findings are important as they establish that the brain of healthy individuals has the capacity to very rapidly experience ownership of a limb amputee's body. Thus, they demonstrate that a full-body illusion can persist also in the context of such substantial visuo-proprioceptive incongruence between the felt and seen body. Moreover, our results show that in this illusory state individuals can experience telescoping, with the felt hand retracting into the seen stump of the artificial body. This is a dramatic example of how the multisensory mechanisms producing ownership of an entire body (Petkova and Ehrsson, 2008; Petkova et al., 2010) influence ownership of a single limb, to the extent of overriding previous notions of visuo-proprioceptive constraints of limb-ownership referring to the fact that the owned object has to resemble a human hand (Tsakiris et al., 2010). In addition to these theoretically relevant aspects, the present results are also important from a clinical perspective as they introduce a new non-invasive method for the potential manipulation of painful telescoped phantom limbs in amputees.

The present findings represent a major advance with respect to earlier studies reporting illusory elongation or shrinking of limbs. In these earlier reports it has been described how perceived arm length can be experimentally manipulated through discordant tactile input to both arms (Craske et al., 1984), or trough muscle tendon vibration (de Vignemont et al., 2005; Longo et al., 2009). In these studies participants always have their eyes closed while conflicting tactile and proprioceptive stimulation was provided to two body parts (or two muscle tendons in the case of Longo et al., 2009). The present study is different as the telescoping occurred while participants experienced a full-body illusion during which they saw an amputated stump, and while the tactile stimulation was only delivered to one body part (their unseen hand). Thus, the mechanisms involved in the current illusory telescoping phenomenon are likely to be different. Unlike the previous experiments, the present telescoping effect results from multisensory processing related to the feeling of ownership of an artificial body, and from the integration of visual information from a seen stump and somatosensory information from ones unseen hand. 


\section{CONSTRAINTS OF BODILY ILLUSIONS}

One theoretically relevant aspect of the current study concerns the constraints of bodily illusions. As mentioned in the introduction, previous studies have demonstrated the malleability of bodily illusions, in the sense that they allow for the artificial body(part) to deviate quite substantially from the real body(part) in shape as well as size (Petkova and Ehrsson, 2008; Haggard and Jundi, 2009; Bruno and Bertamini, 2010). However, to our knowledge this is the first demonstration of a bodily illusion in which the deviation goes as far as a missing limb. In that, it was surprising to observe that all participants experienced the full-body illusion, as shown by the high agreement ratings for the overall body illusion statement in all three experiments. That is, all participants accepted the body of the amputated mannequin as their own, irrespectively of the IH or TH stroking conditions. Although we did not perform a direct comparison with an intact mannequin in the current study, a comparison of the ratings of the current study with those of Petkova and Ehrsson (2008) do not indicate any reduction in the overall full-body illusion strength. The robustness of this illusory experience is a quite remarkable finding in itself. Of course, people who undergo amputations in real life are also put into the situation of suddenly experiencing their body with a missing limb, so in principle the illusion recreates a plausible real life scenario. However, the fact that healthy participants can have such strong illusory sensations of owning an amputated body, while still continuing to "proprioceptively" experience their intact body and sense their own hand, is intriguing.

The strength and robustness of the illusory acceptance of the amputated body is further evidenced if one considers the nature of the stroking in the IH and TH conditions. As described in section "Materials and Methods," in the IH hand condition simultaneous strokes were applied to the end of the mannequin's stump (corresponding to the mannequin's wrist) and the participant's wrist. In contrast, in the TH condition simultaneous strokes were applied end of the mannequin's stump (corresponding to the mannequin's wrist) and the participant's fingertips. In principle, this represents a spatial asynchrony, which is known to disrupt or at least drastically reduce illusory sensations for the RHI (Costantini and Haggard, 2007; Ehrsson et al., 2008). However, for the full-body illusion this did not seem to be the case. How can this be explained? Well, in the context of our full-body illusion, there was spatial congruence between visual, somatosensory and proprioceptive information for all body parts, except the visually missing limb. Consequently, the most likely explanation is that the strength of an overall experienced bodily coherence is sufficient for the illusion to persist even if there is a mismatch for an individual body part. This argument is in line with the findings of Petkova and Ehrsson (2008), who showed that synchronous stimulation of one single body part (i.e., the hand or abdomen) is sufficient to evoke ownership of the entire body. In discussing this finding, the authors refer to the concept of a central body representation consisting of a "map of connected nodes," in which the representations of individual body parts from a continuous whole. In case of sensory conflict regarding one of the nodes, our perceptual systems tend to solve it by producing a single "solution" driven by the priority of maintaining an overall sense of bodily coherence in the best possible way (Lackner, 1988).
In any case, given the robustness of this "hand amputee illusion," an obvious question arises: How far could one take this kind of illusory experience? Firstly, in a "quantitative" sense - how much of the mannequin's body could be missing for one to still accept it as ones own? For example, could the illusion still be induced if the mannequin was missing a whole arm, both arms, etc.? Secondly, in a more "qualitative" sense - does the ease with which the illusion can be induced depend on the specific body part that is missing from the mannequin? For example, would one just as easily accept a body with a missing leg as one with a missing hand? Thirdly, does the amputation of the mannequin have to be physically plausible? That is, what about a scenario in which the mannequin has as a missing or invisible torso? There certainly are plenty of interesting questions to be addressed by future investigations further exploring the constraints of bodily illusions.

\section{PLASTICITY OF BODY REPRESENTATION}

Both the general demonstration of the full-body illusion in the context of a missing limb, as well as the specific demonstration of the illusion of a telescoped limb, make the case for a remarkable degree of plasticity of our body representation. What kind of body representation though? Body representation as such is a loose term, which can refer to different concepts. A distinction often made in the literature is that between body schema and body image (Schwoebel and Coslett, 2004; Kammers et al., 2006, 2010; de Vignemont, 2010). In brief, according to this distinction body schema refers to a dynamic representation of the current position of body parts relative to one another, which is derived from multiple sensory and motor inputs (e.g., visual, tactile, and proprioceptive information), and that interacts with motor systems in the genesis of actions. Body image in contrast refers to the perceptual dimensions of how we experience our body, sometimes referring also to lexical and semantic notions.

There is ongoing controversy in the literature about the exact distinction between, and taxonomy of, different types of body representation (de Vignemont, 2010). While a more detailed discussion of this topic is beyond the scope of the current manuscript, we would nevertheless like to point out that when looked at within this general framework, the results of our study demonstrate plasticity in a number of ways. The results of the pointing task for example, reflecting a change in the sensory proprioceptive representation of the current position of ones hand following an only very short time of experimental manipulation, is likely to reflect plasticity of the body schema. The visual judgment task on the other hand, in which participants had to visually judge the perceived position of their hand with respect to the stump of the mannequin, taps more into body image. Lastly, and in more general terms, the mere fact that healthy participants affirmed to experience an amputated body as their own, shows that even the long-term representation of our body image (consisting of an intact body having two hands and two feet), is not necessarily set in stone at any given moment.

\section{NEURAL CORRELATES OF ILLUSORY TELESCOPING SENSATIONS}

What are the neural mechanisms underlying the illusory experience of a telescoped limb? In recent years, a number of studies have shed light on the neural correlates of bodily illusions and the perceived ownership of artificial body parts or whole bodies. 
In a functional magnetic resonance imaging (fMRI) study, Ehrsson et al. (2004) showed that the RHI is mainly driven by the activity of multisensory areas in intraparietal cortices, as well as by activity in the premotor cortices, which seem to be particularly involved in the self-attribution aspect of the illusion. More recently, Petkova et al. (2010) showed that the same brain regions are also involved in the full-body ownership illusion.

In regard to perceptual changes of the size and shape of body parts, Ehrsson et al. (2005) investigated the neural correlates of the illusory experience of a feeling of shrinking of the waist induced through muscle tendon vibrations. They found that illusory shrinking sensations were associated with activity in higher-order somatosensory areas in the junction between the postcentral and intraparietal sulci, and speculated that similar activations could also be expected for illusory size changes in different body parts. In contrast, the shrinking sensations were not associated with any activity in primary somatosensory or motor areas, which on the other hand seem to be engaged by illusory movements of individual limbs (Naito et al., 2002).

By relating our behavioral findings to these observations, one could speculate that illusory sensations of a telescoped limb could be mediated a combination of these two differential neural mechanisms. That is, while the illusory sensation of shrinking of the arm might be associated with activation in higher-order somatosensory areas, the perceived dislocation of the hand as such could potentially be reflected by a change in activation in primary somatosensory areas. With respect to telescoped phantom limbs in amputees, there is in fact evidence from fMRI investigations that the cortical representation of the phantom in primary somatosensory cortex (S1) changes according to its perceived location. Specifically, it has been shown that imagined movements of a phantom hand generate activation in the areas corresponding to the perceived location of the movement rather than the actual anatomical location of the hand area in S1. That is, imagined movements of nontelescoped phantoms are associated with activity in the S1 region of the hand, partially telescoped phantoms in the region of the arm and completely telescoped phantoms in the region of the shoulder (Flor et al., 2006). Given these findings, the intriguing question arises of whether similar neural mechanisms also underlie illusory telescoping sensations. Of course, while the abovedescribed cortical reorganizations reflect the brain's long-term plasticity following amputation, it remains an empirical question of whether any short-term cortical modulation could be observed in an experimental setting.

\section{THEORETICAL ACCOUNTS OF TELESCOPING IN AMPUTEES}

The experimental setup used in the current study, enabling the induction of illusory telescoping sensations in healthy individuals, could be used to test some aspects of the proposed theoretical accounts of telescoping in amputees. One of these aspects regards the correlation between detail of cortical representation and telescoping speed. As mentioned in the introduction, it has been proposed that telescoping occurs because the proximal portions of the limb are less strongly represented in the cortex relative to the distal portions. In support of this hypothesis, lower limbs seem to telescope more rapidly than upper limbs, supposedly because they are less diffusely represented throughout the cortex due to a lesser involvement in fine motor task (Ramachandran and Hirstein, 1998). A possible way of testing this hypothesis in an experimental setting would be to adopt the full-body illusion as in the current study, but attempt to induce illusory telescoping sensations of upper as well as lower limbs. One could then directly compare the time needed to induce the illusion for different limbs. Under the assumption that the present illusory telescoping in healthy individuals shares some of the mechanisms of telescoping in amputees, one would expect that it takes less time to induce the sensation of a telescoped lower than upper limb.

Secondly, a brief note in regard to the proposed location of shrinking of the phantom limb in amputees. As said above, it has been proposed that it is the proximal part of the limb that shrinks, with a consequently perceived displacement of the distal part (i.e., the phantom hand retracted within the stump). The converging results of our three experiments clearly indicate that participants perceived an actual displacement of their left hand. Moreover, according to questionnaire responses, in Experiment 3 participants also perceived their left arm to be shorter than their right arm. However, on the basis of our current data we cannot conclude whether the perceived displacement of the hand reflects an actual perceived shrinkage of the arm, and if so which exact part of the arm is perceived to shrink. One way of assessing this would be to use the pointing task of the current study, but have participants point to different locations of their arm such as the wrist, elbow, upper arm, etc. This would allow one to investigate whether the proprioceptive drift is restricted to the hand, or whether (and to which extent) would also be observed for other parts of the arm.

A third aspect that could be reproduced experimentally concerns two differential manifestations of telescoping. The most commonly described manifestation of telescoping refers to the displacement of a component of a phantom limb over time, eventually reaching an "end point" (Giummarra et al., 2007). However, in some cases the phenomenon has also been described to occur dynamically, with phantoms rapidly telescoping due to external events such as tactile stimulation of the stump or during active voluntary movements of the phantom (Poeck, 1964). It is likely that the present illusion in healthy individuals shares more common processes with this latter dynamic version of the telescoping phenomenon in amputees. In follow up experiments it would be interesting to investigate this by looking at whether it is possible to actively "pull the invisible hand in and out" within the same stroking session, while the participant keeps viewing the stump of the mannequin. Pilot experiments with members of our lab indeed suggest that this manipulation works very well, but these preliminary findings need to be substantiated in experiments with naïve participants.

\section{CLINICAL RELEVANCE}

To conclude, we would like to discuss one of the potential clinical implications of the current study. As mentioned in the introduction, in amputees telescoping seems to be related to increased levels of phantom pain (Flor et al., 2006), and it has been hypothesized that this is due the mismatch between the sensory feedback from the telescoped phantom and the original somatosensory representation of the hand (Flor et al., 2006). Overall, according to our questionnaire data, the experimentally induced illusion of a telescoped hand was not accompanied by any pain sensations. Nevertheless, given the 
relationship between telescoping and phantom pain in amputees, the intriguing question arises of whether the experimental setup used in our study could be used to investigate the nature of this link, and possibly to manipulate phantom pain sensations. The rationale for postulating the feasibility of such investigations is based on a number of observations from previous literature.

Firstly, with regard to the experimental setup as such, Ehrsson et al. (2008) demonstrated that upper limb amputees can be induced to experience the RHI. That is, following simultaneous stroking of their stump and the finger of the artificial rubber hand, amputees reported strong sensations of touch from the artificial hand. Given this finding, it is plausible to assume that ownership of an artificial hand could also be induced in the context of the full-body illusion. Secondly, with regard to the manipulation of phantom pain sensations, there is evidence for the effectiveness of visual illusions. Specifically, it has been reported that mirror visual feedback (MVF) can be used to alleviate phantom pain (Ramachandran et al., 1995, 2009; Chan et al., 2007; but see Moseley et al., 2008 for a critical review). In brief, during the MVF amputees are put in the position of viewing the reflection of their intact hand in a mirror so that it is optically superimposed on the felt location of their phantom hand. Consequently, movements performed with the intact hand create simultaneous illusory movements of the phantom hand, and such illusory sensations have been found to reduce phantom pain. So, based on the findings that amputees can experience a rubber

\section{REFERENCES}

Botvinick, M., and Cohen, J. (1998). Rubber hands 'feel' touch that eyes see. Nature 391, 756.

Bruno, B., and Bertamini, M. (2010). Haptic perception after a change in hand size. Neuropsychologia 48, 1853-1856.

Carlen, P.L., Wall, P.D., Nadvorna, H., and Steinbach, T. (1978). Phantom limbs and related phenomena in recent traumatic amputations. Neurology 28, 211-217.

Chan, B. L., Witt, R., Charrow, A. P., Magee, A., Howard, R., Pasquina, P. F., Heilman, K. M., and Tsao, J. W. (2007). Mirror therapy for phantom limb pain. N. Engl. J. Med. 357, 2206-2207.

Costantini, M., and Haggard, P. (2007). The rubber hand illusion: sensitivity and reference frame for body ownership. Conscious. Cogn. 16, 229-240.

Craske, B., Kenny, F. T., and Keith, D. (1984). Modifying an underlying component of perceived arm length: adaptation of tactile location induced by spatial discordance. J. Exp. Psychol. Hum. Percept. Perform. 10, 307-317.

de Vignemont, F. (2010). Body schema and body image - pros and cons. Neuropsychologia 48, 669-680.

de Vignemont, F., Ehrsson, H. H., and Haggard, P. (2005). Bodily illusions modulate tactile perception. Curr. Biol. 15, 1286-1290.
Ehrsson, H. H. (2009). How many arms make a pair? Perceptual illusion of having an additional limb. Perception 38, 310-312.

Ehrsson, H. H., Kito, T., Sadato, N., Passingham, R. E., and Naito, E. (2005). Neural substrate of body size: illusory feeling of shrinking of the waist. PLoS Biol. 3, e412. doi: 10.1371/ journal.pbio.0030412

Ehrsson, H. H., Rosén, B., Stockselius, A., Ragnö, C., Köhler, P., and Lundborg, G. (2008). Upper limb amputees can be induced to experience a rubber hand as their own. Brain 131, 3443-3452.

Ehrsson, H. H., Spence, C., and hand! Activity in premotor cortex reflects feeling of ownership of a limb. Science 305, 875-877.

Flor, H., Nikolajsen, L., and Jensen, T. S. (2006). Phantom limb pain: a case of maladaptive CNS plasticity. Nat. Rev. Neurosci. 7, 873-881.

Giummarra, M. J., Gibsonb, S. J., Georgiou-Karistianisa, N., and Bradshawa, J.L. (2007). Central mechanisms in phantom limb perception: the past, present and future. Brain Res. Rev. 54, 219-232.

Haans, A., Ijsselsteijn, W. A., and de Kort, Y. A. (2008). The effect of similarities in skin texture and hand shape on perceived ownership of a fake limb. Body Image 5, 389-394. Passingham, R. E. (2004). That's my

hand as their own, and that visualizing the phantom can reduce phantom pain, it is feasible to hypothesize that the full-body illusion could be used to induce ownership sensations of an intact body in amputees, with the possible consequence of manipulating phantom sensations. The novelty with respect to the MVF technique described above would be that the full-body illusion would allow one to directly manipulate the degree of telescoping of the phantom, and to investigate to which extent this might alter phantom pain sensations.

\section{CONCLUSION}

In sum, our study demonstrates the possibility of experimentally inducing the sensation of a telescoped limb. This finding has a number of theoretical and clinical implications, and sets the scene for a series of potential future studies investigating the plasticity of body representation in healthy individuals as well as phantom sensations in amputees.

\section{ACKNOWLEDGMENTS}

Thanks to: All the participants; Everyone in H. Henrik Ehrsson's lab for their input, especially Alexander Skoglund for technical assistance and Andreas Kalckert for invaluable discussions and feedback; This work was funded by the Swedish Foundation for Strategic Research, the European Research Council, and the Human Frontiers of Science Program.

Haggard, P., and Jundi, S. (2009). Rubber hand illusions and size-weight illusions: self-representation modulates representation of external objects. Perception 38, 1796-1803.

Kammers, M. P., Kootker, J. A., Hogendoorn, H., and Dijkerman, $\mathrm{H}$ C. (2010). How many motoric body representations can we grasp? Exp. Brain Res. 202, 203-212.

Kammers, M. P., van der Ham, I. J., and Dijkerman, H. C. (2006). Dissociating body representations in healthy individuals: differential effects of a kinaesthetic illusion on perception and action. Neuropsychologia 44 2430-2436.

Lackner, J. R. (1988). Some proprioceptive influences on the perceptual representation of body shape and orientation. Brain 111, 281-297.

Longo, M. R., Kammers, M. P. M., Gomi, H., Tsakiris, M., and Haggard, P. (2009). Contraction of body representation induced by proprioceptive conflict. Curr. Biol. 19, R727-R728.

Melzack, R., Isreal, R., Lacroix, R., and Schultz, G. (1997). Phantom limbs in people with congenital limb deficiency or amputation in early childhood. Brain 120, 1603-1620.

Moseley, G. L., Gallace, A., and Spence, C. (2008). Is mirror therapy all it is cracked up to be? Current evidence and future directions. Pain 138, 7-10.
Naito, E., Roland, P. E., and Ehrsson, H. H. (2002). I feel my hand moving: a new role of the primary motor cortex in somatic perception of limb movement. Neuron 36, 979-988.

Oldfield, R. C. (1971). The assessment and analysis of handedness: the Edinburgh inventory. Neuropsychologia 9, 97-114.

Petkova, V. I., and Ehrsson, H. H. (2008). If I were you: perceptual illusion of body swapping. PLoS ONE 3:e3832. doi: 10.1371/journal.pone.0003832

Petkova, V. I., Gentile, G., Khoshnevis, M., Jonsson, T., Li, Q., and Ehrsson, H.H. (2010). "My perspective matters: the feeling of ownership of an entire body depends on the visual firstperson perspective," in Neuroscience Meeting Planner (San Diego, CA: Society for Neuroscience). Available at: http://www.abstractsonline.com/ Plan/SSResults.aspx

Poeck, K. (1964). Phantoms following amputation in early childhood and in congenital absence of limbs. Cortex 1, 269-275.

Ramachandran, V. S., Brang, D., and McGeoch, P.D. (2009). Size reduction using mirror visual feedback (MVF) reduces phantom pain. Neurocase 15, 357-360.

Ramachandran, V. S., and Hirstein, W. (1998). The perception of phantom limbs: the D.O. Hebb lecture. Brain 121, 1603-1630. 
Ramachandran, V. S., RogersRamachandran, D., and Cobb, S. (1995). Touching the phantom limb. Nature 377, 489-490.

Schwoebel, J., and Coslett, H. B. (2004). Evidence for multiple, distinct representations of the human body.J. Cogn. Neurosci. 17, 543-553.

Tsakiris, M., Carpenter, L., James, D., and Fotopoulou, A. (2010). Hands only illusion: multisensory integration elicits sense of ownership for body parts but not for non-corporeal objects. Exp. Brain Res. 204, 343-352. Tsakiris, M., and Haggard, P. (2005). The rubber hand illusion revisited: visuotactile integration and self-attribution. J. Exp. Psychol. Hum. Percept. Perform. 31, 80-91.

Conflict of Interest Statement: The authors declare that the research was conducted in the absence of any commercial or financial relationships that could be construed as a potential conflict of interest.

Received: 25 December 2010; accepted: 21 March 2011; published online: 01 April 2011.

Citation: Schmalzl L and Ehrsson $\mathrm{HH}$ (2011) Experimental induction of a perceived "telescoped" limb using a full- body illusion. Front. Hum. Neurosci. 5:34. doi: 10.3389/fnhum.2011.00034

Copyright (c) 2011 Schmalzl and Ehrsson. This is an open-access article subject to a non-exclusive license between the authors and Frontiers Media SA, which permits use, distribution and reproduction in other forums, provided the original authors and source are credited and other Frontiers conditions are complied with. 